\title{
Stereoselective Total Synthesis of (+)-Streptazolin by Using a Temporary Silicon-tethered RCM Strategy
}

\author{
Fangzheng Li, Marvin J. Miller* \\ Department of Chemistry and Biochemistry \\ University of Notre Dame, Notre Dame, IN 46556 \\ mmiller1@nd.edu
}

\section{Supporting Information: Table of Content}

General Experimental Methods and Notes $\quad$ S2

${ }^{1} \mathrm{H}$ NMR Spectrum of $\mathbf{1 1}$ S3

${ }^{13}$ C NMR Spectrum of $\mathbf{1 1} \quad$ S4

${ }^{1} \mathrm{H}$ NMR Spectrum of $\mathbf{1 9} \quad$ S5

${ }^{13} \mathrm{C}$ NMR Spectrum of $\mathbf{1 9}$ S6

${ }^{1} \mathrm{H}$ NMR Spectrum of $\mathbf{2 0} \quad$ S7

${ }^{13} \mathrm{C}$ NMR Spectrum of $\mathbf{2 0}$ S8

${ }^{1} \mathrm{H}$ NMR Spectrum of $\mathbf{2 1}$ S9

$\begin{array}{lr}{ }^{13} \mathrm{C} \text { NMR Spectrum of } \mathbf{2 1} & \text { S10 }\end{array}$

${ }^{1} \mathrm{H}$ NMR Spectrum of $22 \quad$ S11

${ }^{13}$ C NMR Spectrum of $22 \quad$ S12

${ }^{1} \mathrm{H}$ NMR Spectrum of $\mathbf{2 5} \quad \mathrm{S} 13$

$\begin{array}{lr}{ }^{13} \text { C NMR Spectrum of } 25 & \text { S14 }\end{array}$

${ }^{1} \mathrm{H}$ NMR Spectrum of $\mathbf{2 6} \quad \mathrm{S} 15$

${ }^{13} \mathrm{C}$ NMR Spectrum of $\mathbf{2 6} \quad \mathrm{S} 16$

${ }^{1} \mathrm{H}$ NMR Spectrum of $\mathbf{2 7} \quad$ S17

$\begin{array}{lr}{ }^{13} \mathrm{C} \text { NMR Spectrum of } \mathbf{2 7} & \mathrm{S} 18\end{array}$

${ }^{1}$ H NMR Spectrum of $\mathbf{2 9} \quad$ S19

$\begin{array}{lr}{ }^{13} \text { C NMR Spectrum of } 29 & \text { S20 }\end{array}$

${ }^{1} \mathrm{H}$ NMR Spectrum of $\mathbf{3 0} \quad$ S21

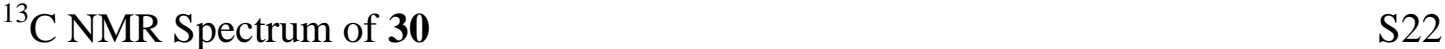

$\begin{array}{ll}{ }^{1} \mathrm{H} \text { NMR Spectrum of } \mathbf{3 1} & \text { S23 }\end{array}$

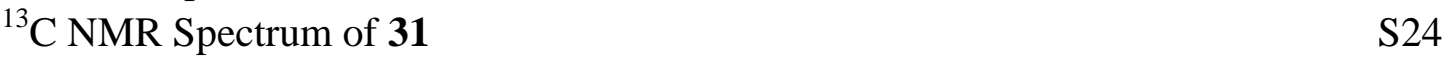

$\begin{array}{lr}{ }^{1} \mathrm{H} \text { NMR Spectrum of } \mathbf{3 2} & \text { S25 }\end{array}$

$\begin{array}{lr}{ }^{1} \mathrm{H} \text { NMR Spectrum of } \mathbf{1} & \text { S26 }\end{array}$

${ }^{13}$ C NMR Spectrum of $\mathbf{1} \quad$ S27

${ }^{1} \mathrm{H}$ NMR Spectrum of the crude mixture of (+)-1 and its E-isomer (J. Org. Chem. 2004, 69, 8836) S28

${ }^{13} \mathrm{C}$ NMR Spectrum of the crude mixture of $(+)-\mathbf{1}$ and its E-isomer (J. Org. Chem. $\begin{array}{ll}2004,69,8836) & \text { S29 }\end{array}$

${ }^{1}$ H NMR Spectrum of (+)-2 $\quad$ S30

${ }^{13}$ C NMR Spectrum of $(+)-2 \quad$ S31 
General Methods and Notes: Melting points were uncorrected. ${ }^{1} \mathrm{H}$ and ${ }^{13} \mathrm{C}$ NMR spectra were obtained on a 300, 500 or 600 spectrometer. Infrared spectra were recorded on a TFIR, and TF refers to thin film. All specific optical rotation was measured on at $546 \mathrm{~nm}$ and $20{ }^{\circ} \mathrm{C}$. Analytical TLC was carried out using aluminum-backed $0.2 \mathrm{~mm}$ silica gel 60 F-254 plates. Column chromatography was conducted using silica gel 60 (230-400) meshes. All reactions were periodically monitored by TLC and worked up after the complete consumption of starting materials unless specified otherwise. All purchased reagents were of reagent grade quality and were used without further purification.

Since we found that compounds 19-27, 29-32, 1, tend to partially decompose when concentrated under high vaccum to remove of trace of solvents and water. NMR of those compounds was taken without or subsequent to a short time (15 minutes) of high vacuum concentration. ${ }^{1} \mathrm{H}$ and ${ }^{13} \mathrm{C}$ NMR peaks of compounds 29, 30 , 31 and 32 were found relative weaker and broader due to the existence of rotamers of the carbamate unit in those molecules. ${ }^{13} \mathrm{C}$ NMR of compound 32 was not obtained because of this rotamer phenomenon and its tendency to decompose. 


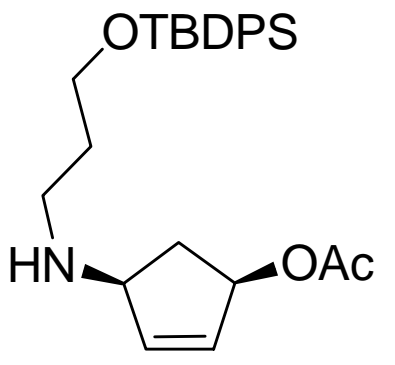

11

${ }^{1} \mathrm{H}$ NMR $\left(300 \mathrm{MHz}, \mathrm{CDCl}_{3}\right)$

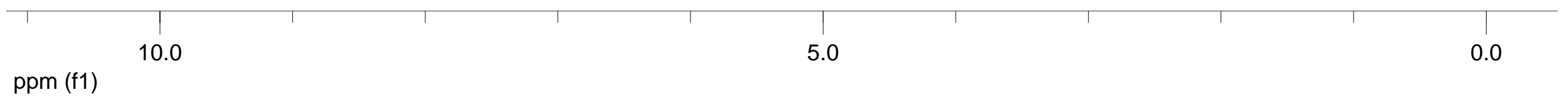




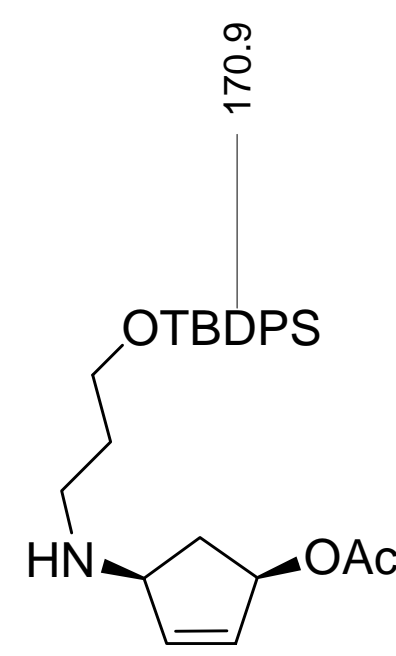

11

${ }^{13} \mathrm{C}$ NMR $\left(75 \mathrm{MHz}, \mathrm{CDCl}_{3}\right)$
๑

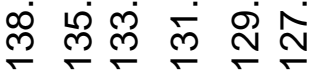

$\stackrel{\circ}{\infty}$

กิن

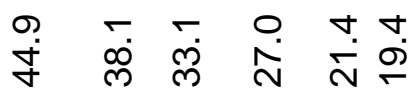

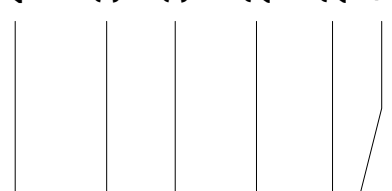

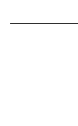

ppm (f1) 


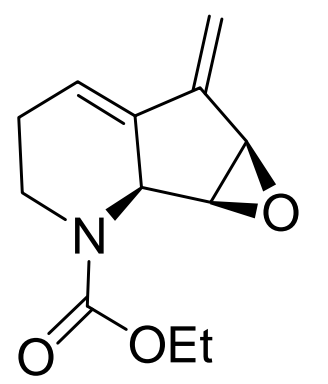

19

${ }^{1} \mathrm{H}$ NMR $\left(500 \mathrm{MHz}, \mathrm{CDCl}_{3}\right)$ 


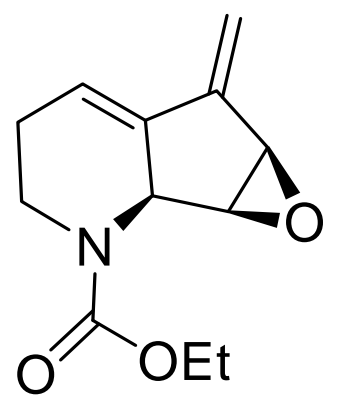

19

${ }^{13} \mathrm{C}$ NMR $\left(125 \mathrm{MHz}, \mathrm{CDCl}_{3}\right)$

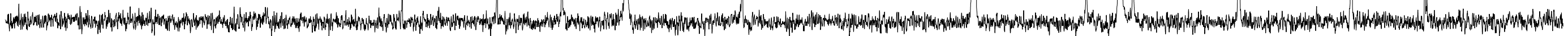

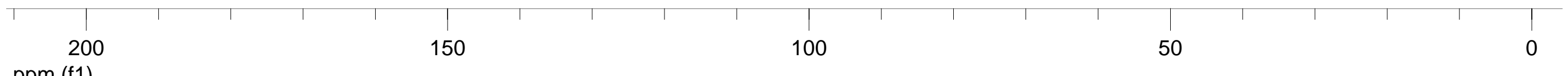




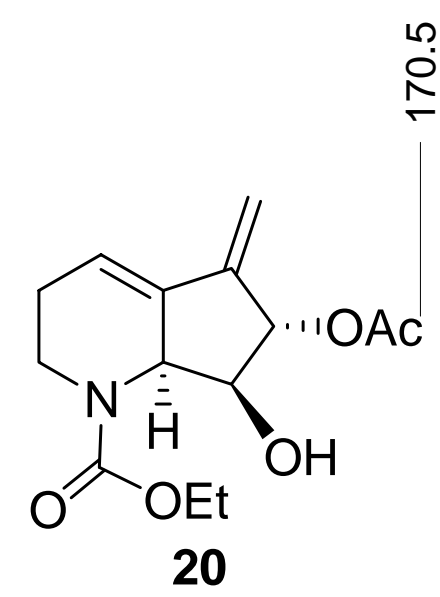

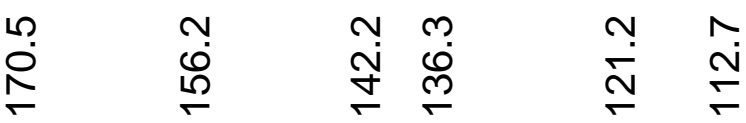

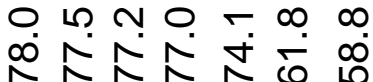

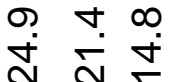

${ }^{13} \mathrm{C}$ NMR $\left(125 \mathrm{MHz}, \mathrm{CDCl}_{3}\right)$

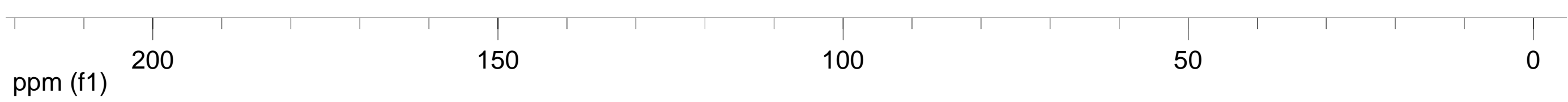




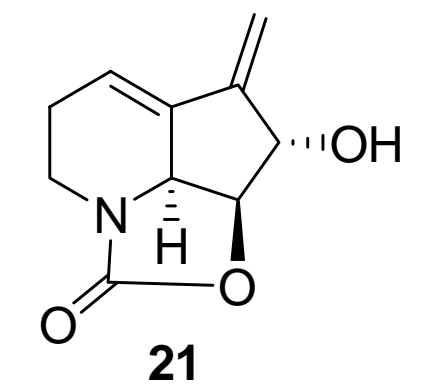

${ }^{1} \mathrm{H}$ NMR $\left(500 \mathrm{MHz}, \mathrm{CDCl}_{3}\right.$, trace of EtOAc)

10.0 ppm (f1) 


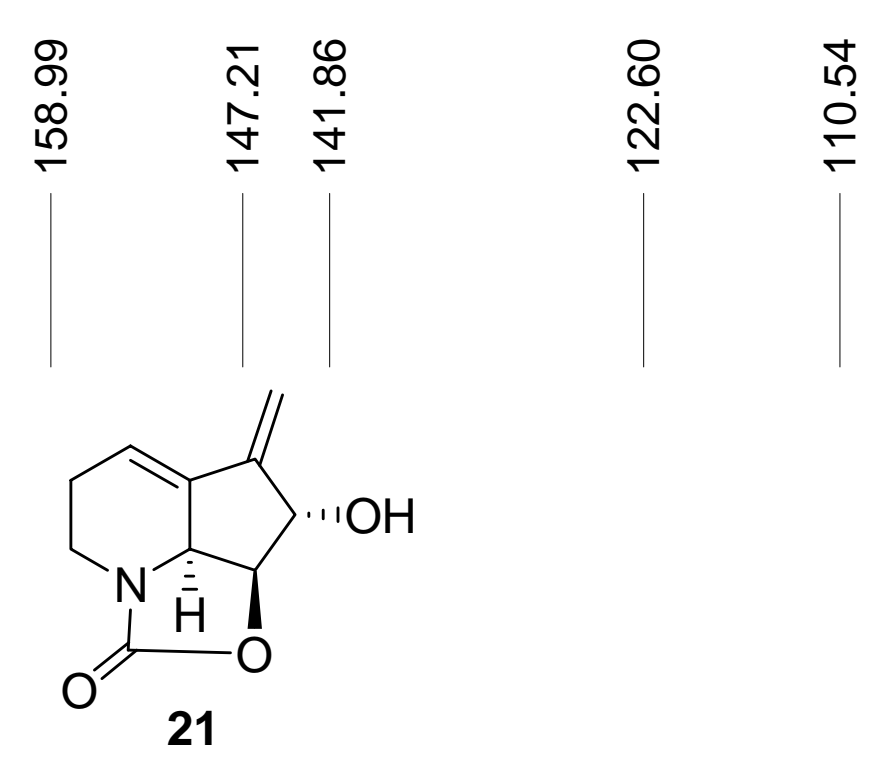

${ }^{13} \mathrm{C} \mathrm{NMR}\left(125 \mathrm{MHz}, \mathrm{CDCl}_{3}\right)$

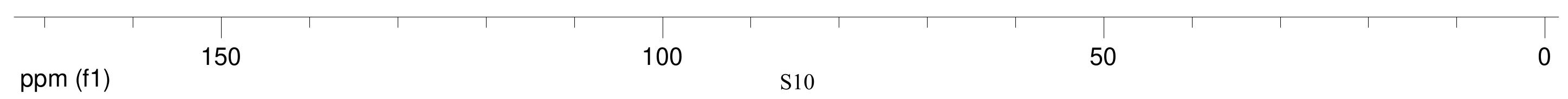




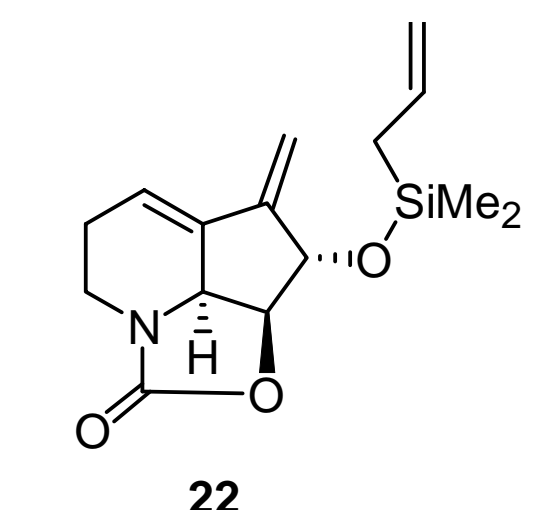

${ }^{1} \mathrm{H}$ NMR $\left(500 \mathrm{MHz}, \mathrm{CDCl}_{3}\right)$

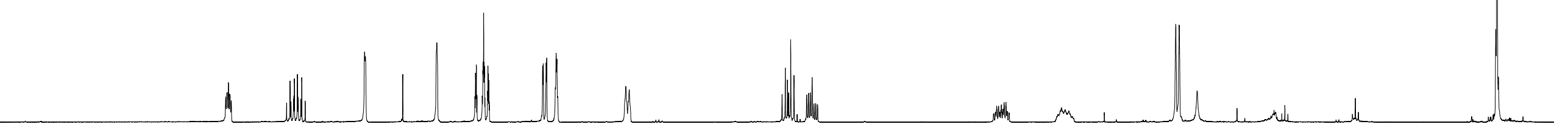




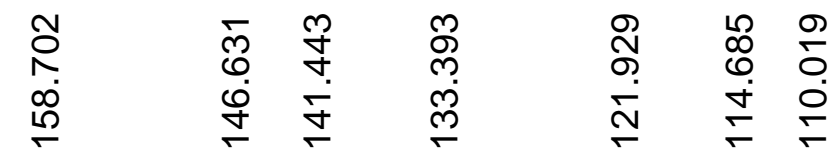

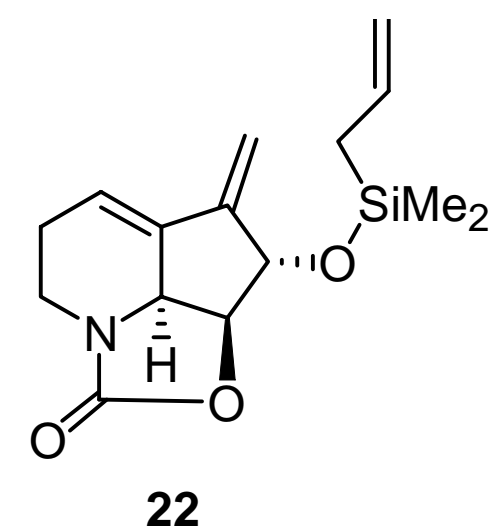

${ }^{13} \mathrm{H}$ NMR (150 MHz, CDCl 3 )

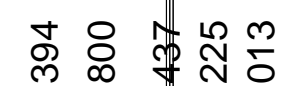

i

\&)

กิ

암

ले

ำ

ํํํ 


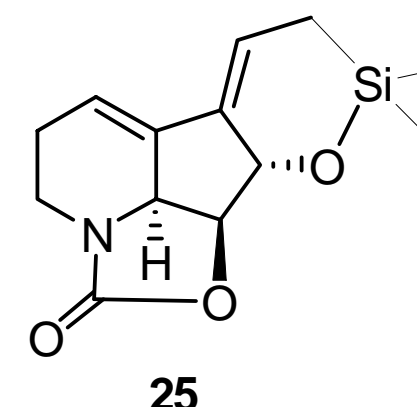

25

${ }^{1} \mathrm{H}$ NMR $\left(500 \mathrm{MHz}, \mathrm{CDCl}_{3}\right)$

$$
8.0
$$

5.0 

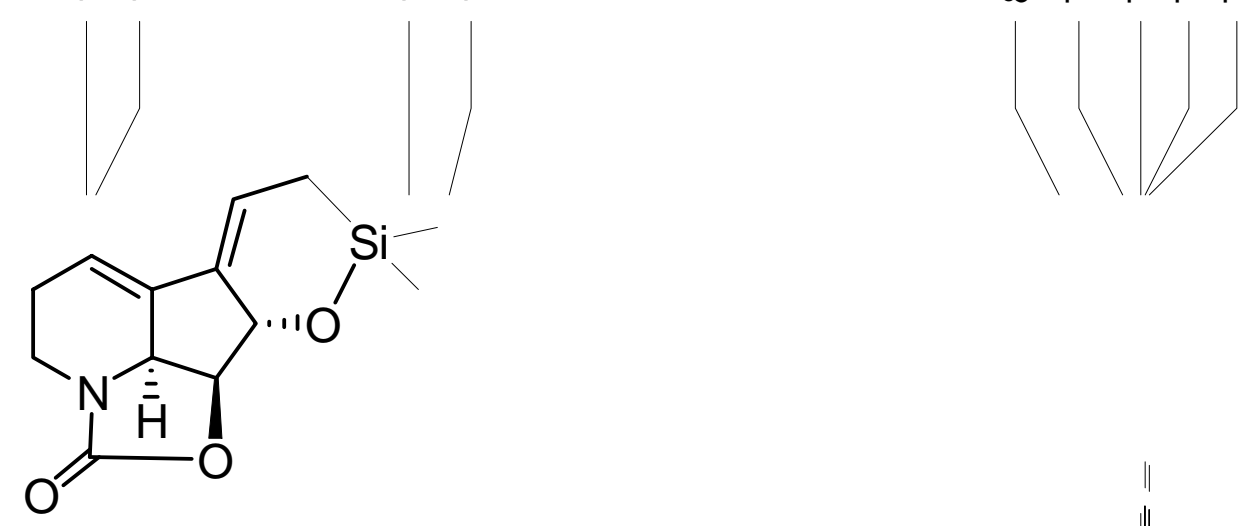

\section{5}

${ }^{13} \mathrm{H}$ NMR (125 MHz, CDCl 3 ) 


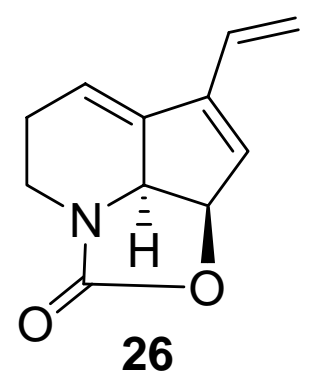

${ }^{1} \mathrm{H}$ NMR $\left(500 \mathrm{MHz}, \mathrm{CDCl}_{3}\right)$

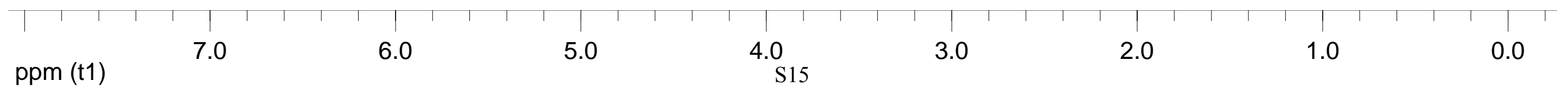




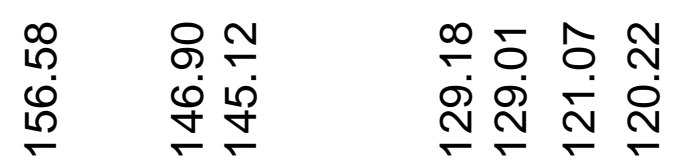
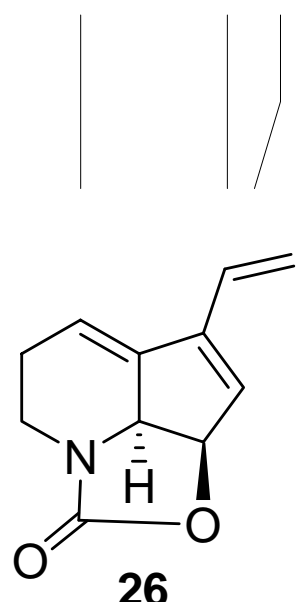

${ }^{13} \mathrm{C}$ NMR $\left(125 \mathrm{MHz}, \mathrm{CDCl}_{3}\right)$

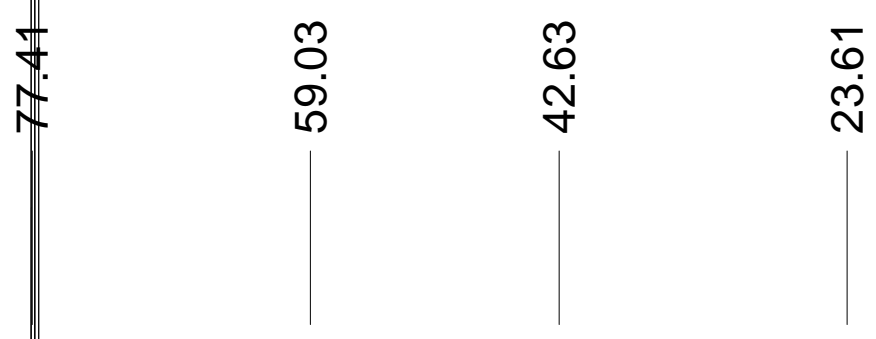




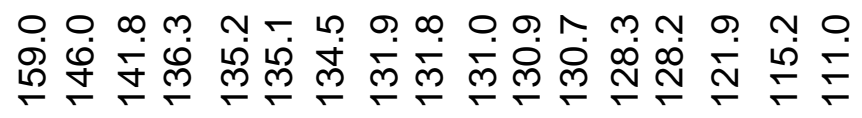

무

$\begin{array}{ll}\forall & 0 \\ 0 & 0 \\ 0 & 0\end{array}$

ᄂ?

ํㅗํ

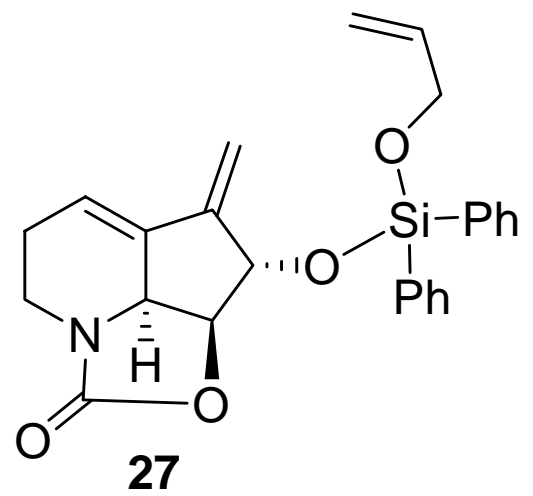

${ }^{13} \mathrm{C}$ NMR $\left(125 \mathrm{MHz}, \mathrm{CDCl}_{3}\right)$

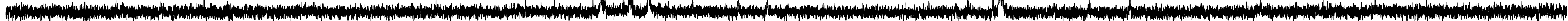

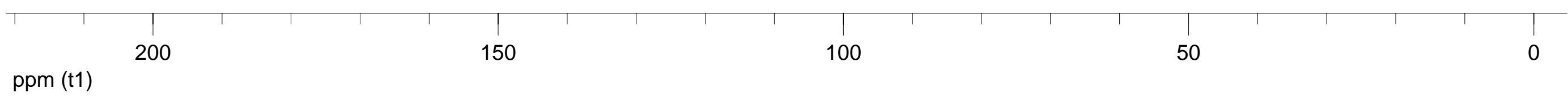




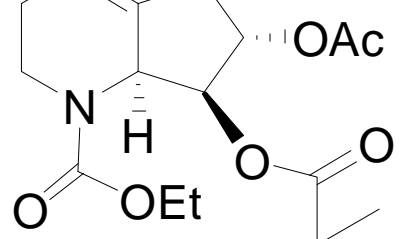

29

${ }^{1} \mathrm{H}$ NMR (300 MHz, CD $\left.{ }_{3} \mathrm{OD}\right)$ 


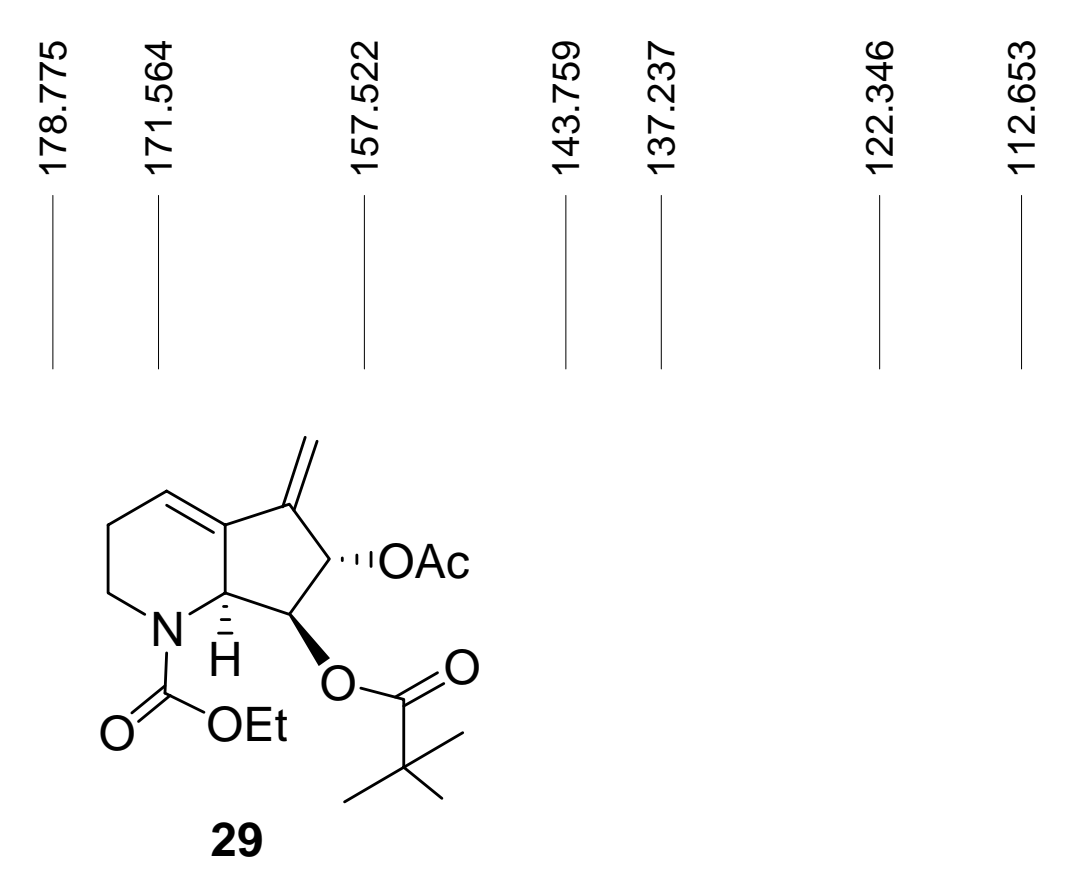

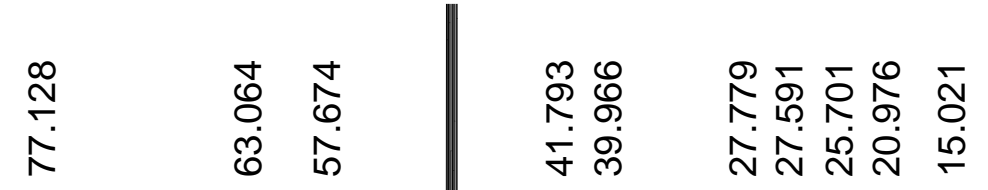

${ }^{13} \mathrm{H}$ NMR $\left(125 \mathrm{MHz}, \mathrm{CD}_{3} \mathrm{OD}, 50^{\circ} \mathrm{C}\right)$ 


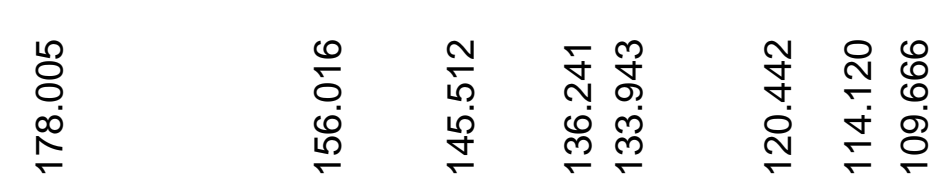
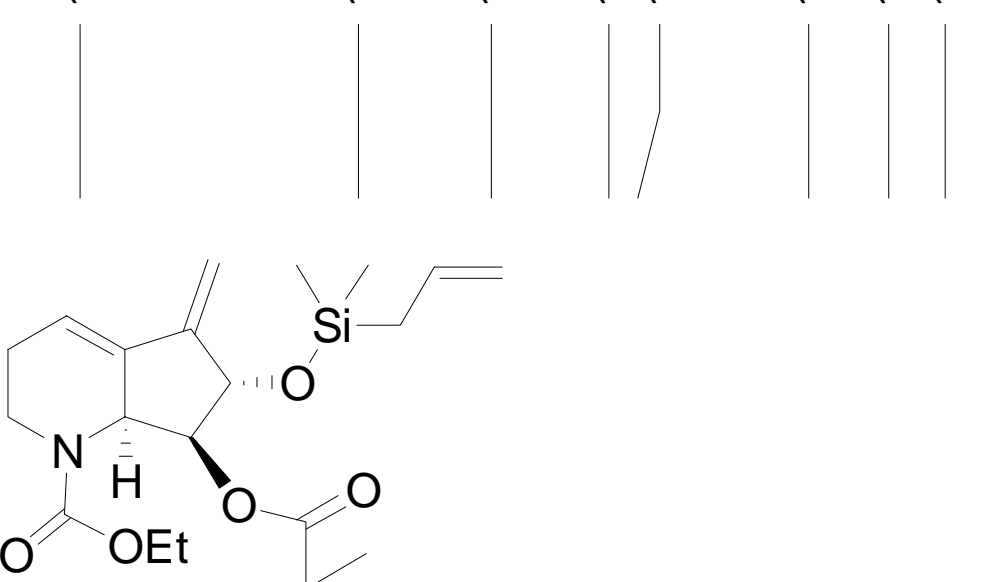

30

${ }^{13} \mathrm{H}$ NMR $\left(150 \mathrm{MHz}, \mathrm{CDCl}_{3}\right)$

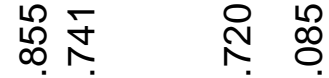

命

$\overline{\overline{0}}$

勇

守

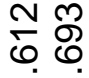




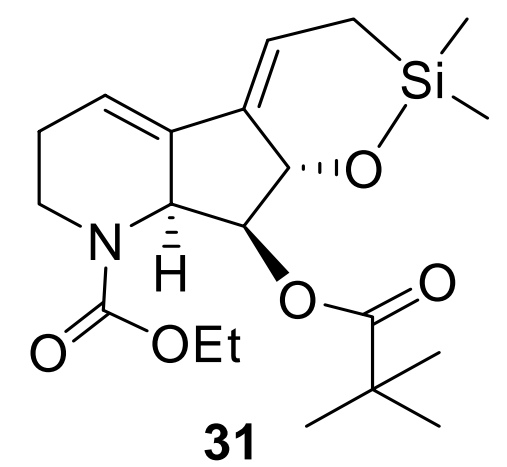

${ }^{1} \mathrm{H} \mathrm{NMR}\left(300 \mathrm{MHz}, \mathrm{CDCl}_{3}\right)$ 


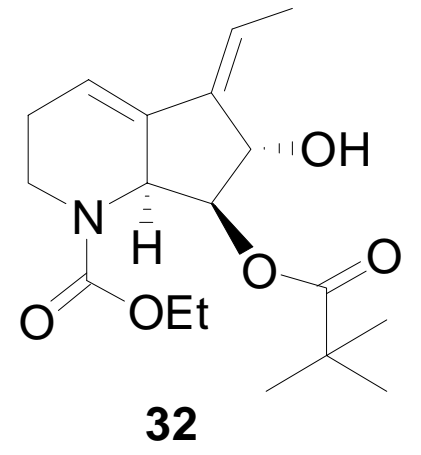

${ }^{1} \mathrm{H}$ NMR $\left(300 \mathrm{MHz}, \mathrm{CDCl}_{3}\right)$ 


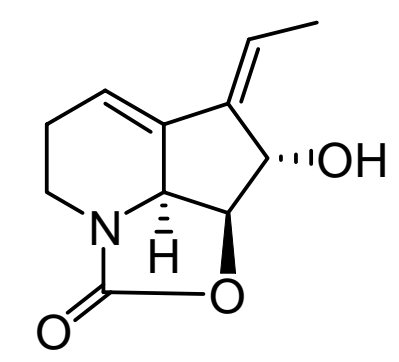

( + )-Streptazolin 1

${ }^{1} \mathrm{H}$ NMR $\left(300 \mathrm{MHz}, \mathrm{CDCl}_{3}\right)$ 


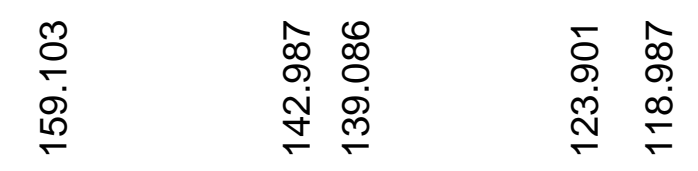

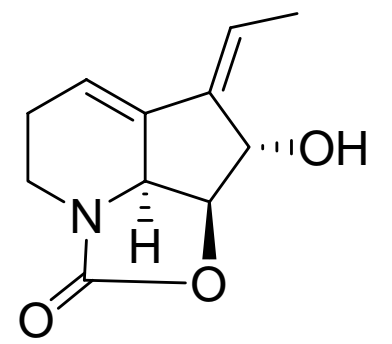

( + )-Streptazolin 1

$\left.{ }^{13} \mathrm{C} \mathrm{NMR} \mathrm{(125} \mathrm{MHz,} \mathrm{CDCl}_{3}\right)$

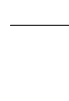




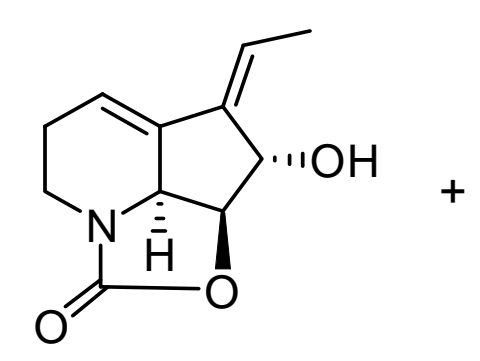

( + )-Streptazolin 1

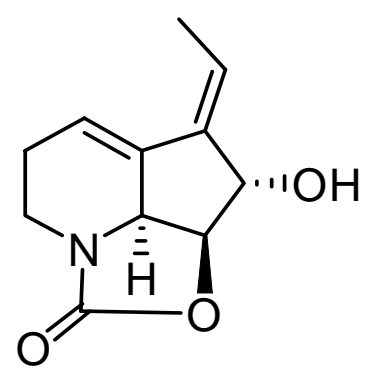

E-isomer

${ }^{1} \mathrm{H}$ NMR (500 MHz, $\mathrm{CDCl}_{3}$ ) J. Org. Chem. 2004, 69, 8836

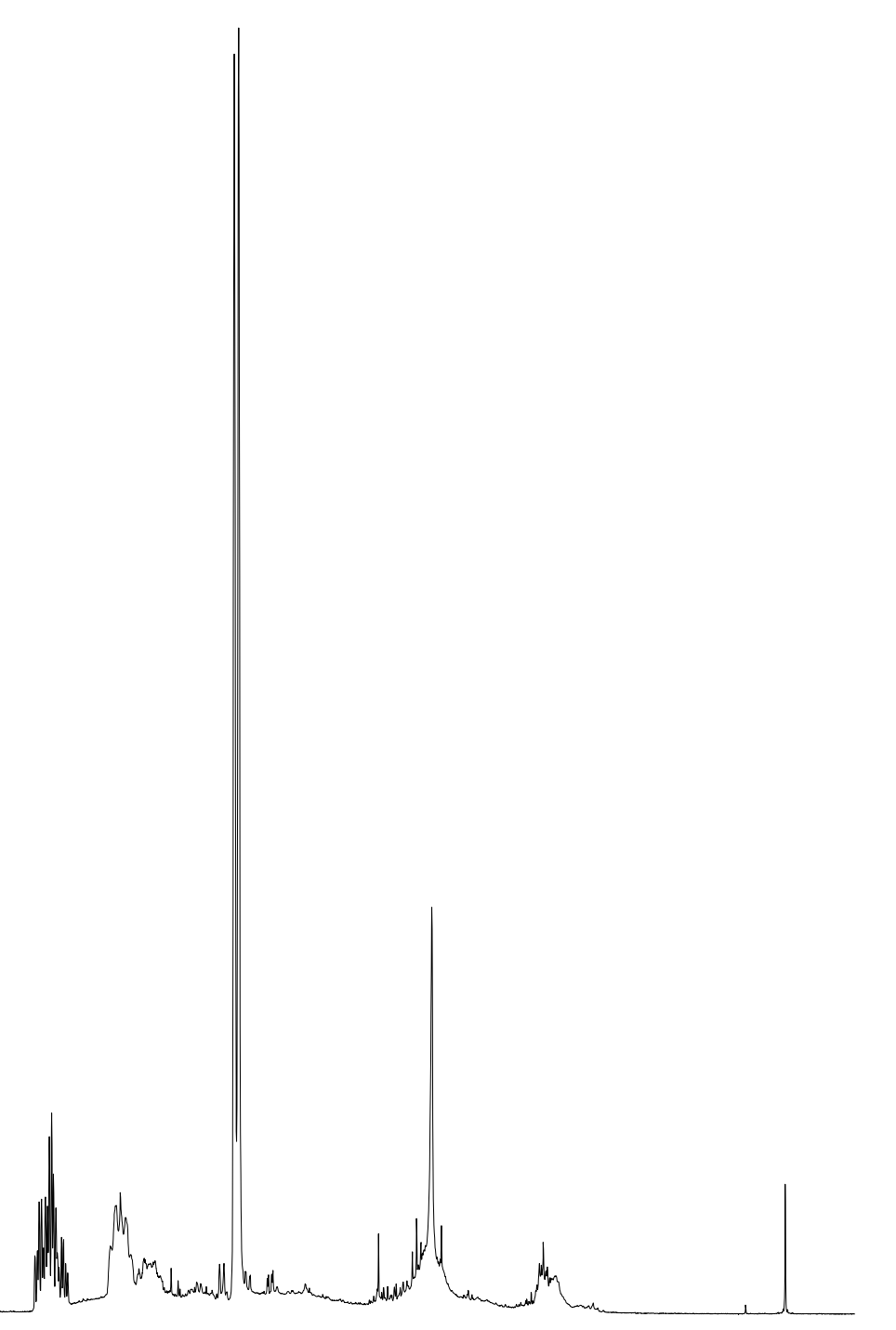

8.0

6.0

5.0

3.0

2.0

1.0 


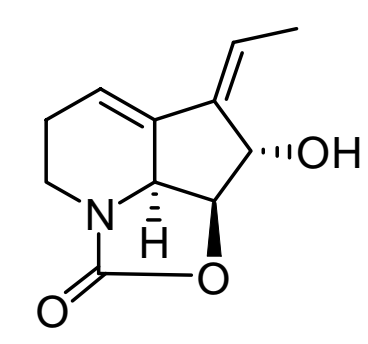

( + )-Streptazolin 1

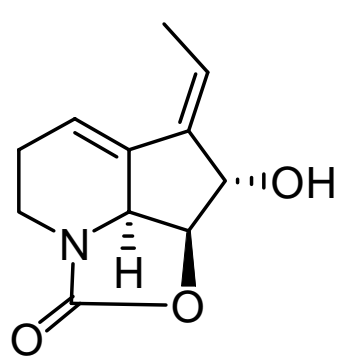

E-isomer

${ }^{1} \mathrm{C}$ NMR (125 MHz, $\left.\mathrm{CDCl}_{3}\right)$ J. Org. Chem. 2004, 69, 8836 


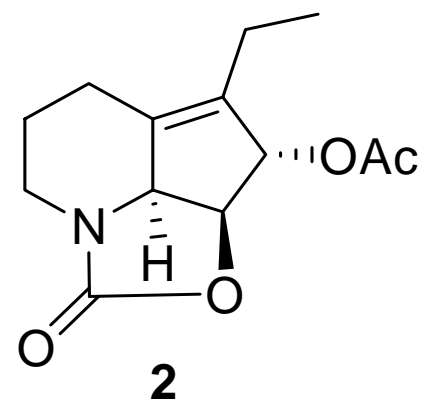

${ }^{1} \mathrm{H}$ NMR $\left(600 \mathrm{MHz}, \mathrm{CDCl}_{3}\right)$

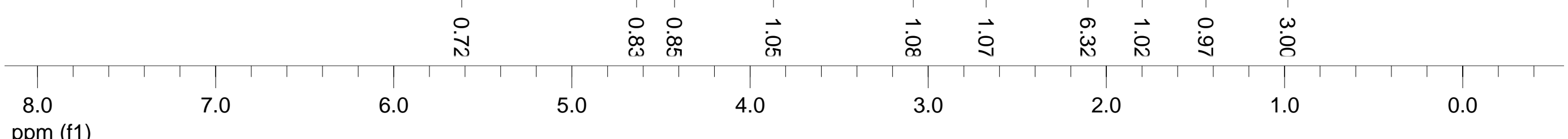




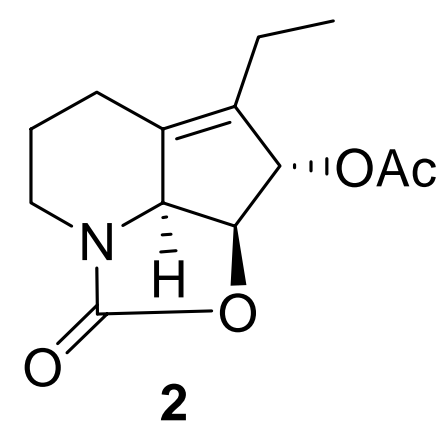

${ }^{13} \mathrm{C}$ NMR $\left(150 \mathrm{MHz}, \mathrm{CDCl}_{3}\right)$ 